\title{
Lipid-Based Membrane Domains: Physics Meets Immunology
}

\author{
Thomas Harder ${ }^{1, *}$ and Gerrit van Meer ${ }^{2}$ \\ ${ }^{1}$ Sir William Dunn School of Pathology, South Parks Road, \\ Oxford OX1 3RE, UK \\ 2 Department of Membrane Enzymology, Institute of \\ Biomembranes, Utrecht University, Padualaan 8, $3584 \mathrm{CH}$ \\ Utrecht, the Netherlands \\ * Corresponding author: Thomas Harder, \\ thomas.harder@path.ox.ac.uk
}

Received 14 July 2003, revised and accepted for publication 24 July 2003

Biological activities ranging from signaling, membrane transport to pathogenic processes take place in segregated subdomains of cell membranes. A fundamental step towards understanding the organization of membrane domains arose from the raft hypothesis, which proposes that these processes occur in raft membrane domains formed by lateral lipid assemblies enriched in sphingolipid and cholesterol (1).

For many years detergent-resistant membranes were used as an operational definition for raft membrane domains and were an essential tool for studying raft composition and protein behavior. Further efforts were made to define the correlation between rafts/detergentresistant membranes in cells and physical properties of membrane phases described in artificial bilayers (2).

Accordingly, the prediction is that the membrane is a mosaic of areas in which the lipids are fluid but display 'phases' with different physical properties and chemical composition. Each membrane protein will have an intrinsic preference for one of the areas. The evidence for lipidbased domains, their size and dynamics, and their biological function were the subject of intense debate at the recent Euroconference on Lipid Rafts in Tomar, Portugal, held May 17-22, 2003.

\section{How Biophysicists View Membrane Phases}

It is now generally accepted that the occurrence of lipid domains in biomembranes is caused by lipid-lipid immiscibility. Whereas the lateral separation of lipids into a solid gel phase and a fluid liquid crystalline phase has been studied since the early 1970s, a breakthrough in this field was the realization in the 1980s that phospholipids and cholesterol can form two (or more) immiscible fluid phases within the same membrane (3). This development coincided with the realization in cell biology that cells need to separate lipids to generate the different lipid compositions of the various organelle membranes (4) and the definition of the lipids involved.

Winchill Vaz (Coimbra) and Manuel Prieto (Lisbon) described techniques that are being used to determine the presence of lipid phases in model membranes. Manuel described the phase-diagram of the mixture of palmitoyl sphingomyelin (SM), palmitoyloleoyl phosphatidylcholine (PC) and cholesterol, representing the three most abundant lipid types in the outer leaflet of mammalian plasma membranes (Figure 1). The phase-diagram shows the occurrence of the various phases for each possible ratio of the three components. In the range of $30-40 \mathrm{~mol} \%$ cholesterol and in the presence of at least $10 \mathrm{~mol} \% \mathrm{PC}$ (values typical for plasma membranes), the mixture displays the coexistence of two liquid-crystalline phases, the more fluid liquid-disordered state and the liquid-ordered state. The phase-diagram also yields information concerning the composition of each of the two phases. In the equimolar mixture $(1: 1: 1)$ and at $37^{\circ} \mathrm{C}$ the liquiddisordered phase consists of $69-57 \%$ PC, 18-33\% SM and $13-10 \%$ cholesterol, while the ordered phase consists of $14-19 \%$ PC, $42-33 \%$ SM and $44-48 \%$ cholesterol. Lowering the temperature to $23^{\circ} \mathrm{C}$ resulted in the appearance of a solid phase above $30-40$ mol\% of SM, which would be high for a biomembrane (Figure 1).

Paavo Kinnunen (Helsinki) warned against the uncritical application of terms derived from physical measurements on model membranes to the situation in living cells. The lateral and transversal lipid composition of biomembranes with some 1000 different types of lipids is highly complex and cannot be adequately described. He also warned against simplified descriptions of what causes the clustering of sphingolipids and cholesterol like van der Waals interactions or hydrogen bonding, and illustrated how the hydrophobic cholesterol favorably packs under the umbrella of a sphingolipid headgroup that has a larger cross-sectional area than its lipid tails, with the two molecules having phospholipids (5). Along the same line, Winchill Vaz mentioned that not only SM and disaturated PC but also monounsaturated PC to some extent favorably 


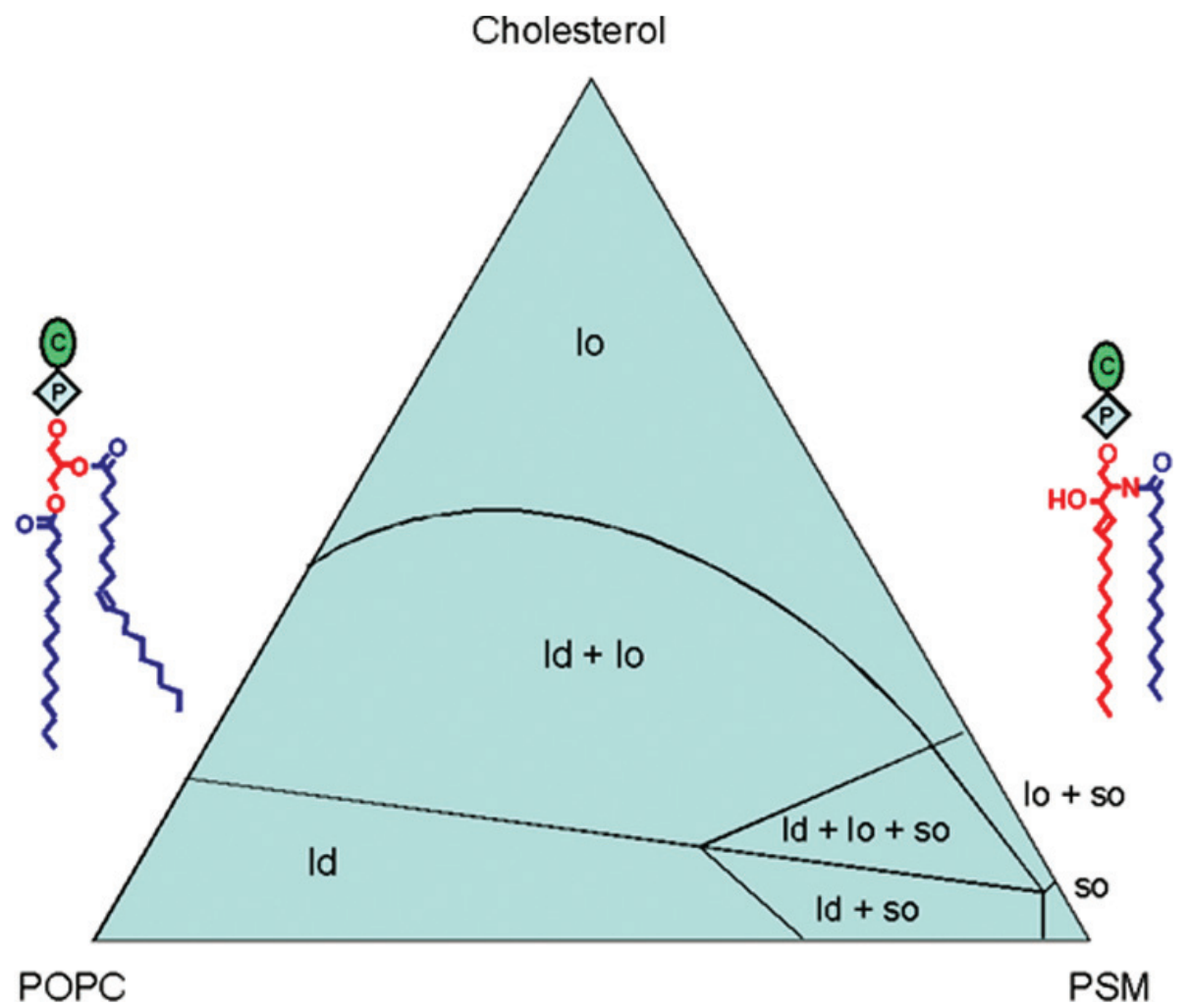

Figure 1: Phase diagram at $37^{\circ} \mathrm{C}$ of a lipid mixture consisting of phosphatidylcholine containing a palmitate (C16:0) and an oleate (C18:1) chain (POPC), sphingomyelin containing a palmitate (PSM), and cholesterol. Each corner of the triangle represents a pure lipid. The top is pure cholesterol, near which the lipid bilayer is in a liquid-ordered (lo) phase; the bottom left is pure POPC in a liquid-disordered (Id) phase; and the bottom-right represents PSM in a solid-ordered (so) phase. The baseline connecting POPC with PSM represents lipid mixtures starting from POPC and containing an increasing fraction of PSM. This is the same for each line parallel to the baseline that can be drawn in the triangle. However, the mixtures on the baseline contain no cholesterol, and the higher the line is drawn in the triangle, the higher the cholesterol concentration. Each point in the triangle defines one specific ratio of the three lipids. The $1: 1: 1$ ratio is situated right in the middle. It displays both the $\mathrm{ld}+\mathrm{lo}$ phases [adapted from: de Almeida RFM, Fedorov $A$ and Prieto $M$. (2003) Biophys J., in press]. interact with cholesterol, as is clear from the condensing effect on such a lipid.

The occurrence of planar domains is energetically unfavorable for a membrane because the two phases have a different spontaneous curvature. Madan Rao (Bangalore) illustrated how the chirality of cholesterol may induce a membrane deformation, resulting in vesicle budding (6). One other energetically unfavorable factor is the line tension at the interphase boundary, which is proportional to the length of that boundary. The chirality-curvature coupling, the spontaneous curvature and the line tension (7) provide a driving force for budding, resulting in spherical, flaskshaped or grape-like invaginations (6). The spontaneous curvature of a domain would be zero if similar domains occurred on both sides of the bilayer. Gerrit van Meer (Utrecht) described how the activity of a family of lipid translocases in the cholesterol-rich membranes of Golgi, endosome and plasma membrane may be one driving force behind vesicle budding from those membranes (8). At the same time, Jean Gruenberg (Geneva) described how a low internal $\mathrm{pH}$ in addition to the presence of the special phospholipid lysobisphosphatidic acid (LBPA) results in a spontaneous inward budding in liposomes, possibly explaining how multivesicular endosomes arise by inward budding in the LBPA-containing and acidic late endosomes.

\section{Raft Domains: Structure of the Untouched Plasma Membrane}

Therefore, it is the central challenge for raftologists to understand how the concept of phase separation, firmly established in artificial membrane systems, can be transferred to model the structure of the cell's plasma membrane bilayer.

Ken Jacobson (Chapel Hill) showed the partitioning of proteins and lipids into separate raft-like ordered membrane domains that form in glass-supported artificial lipid monolayers. He found that the neuronal myristoylated protein NAP-22 partitioned into GM1-enriched membrane domains. However, when this observation was transferred to the cell, fluorescence microscopy of NAP-22 and GM1 showed only partial organization into overlapping membrane domains (9). This observation reflects the dilemma described by many researchers over the years: by most microscopic techniques, cohesion between non-cross-linked raft markers are difficult to detect. This has been accounted for by the small size and dynamic nature of raft domains in plasma membranes. Ken Jacobson put forward a concept of stable shells of 50-100 lipid molecules that surround specific proteins giving them an affinity for ordered membrane phases. Possibly such a 


\section{Harder et al.}

shell can target proteins into larger membrane domains (for example, caveolae) upon association and/or cross-linking by a ligand (10).

Jitu Mayor (Bangalore) utilized fluorescence resonance energy transfer (FRET) between different GPI-anchored proteins to assay clustering of GPI-anchored proteins as detected by their homo-FRET methodology outlined earlier (11). Based on dilution and bleaching experiments, Jitu Mayor estimated that GPl-anchored protein clusters are very small, containing few receptor molecules, while many receptor molecules may actually be monomeric. They also showed clustering between the diverse GPI-anchored protein molecules. Further, antibody-mediated clustering of a specific $\mathrm{GPI}$-anchored protein reorganizes the nanoscale organization of native GPI-anchored protein clusters.

Ben Nichols (Cambridge, UK) studied the plasma membrane organization of GM1 ganglioside using energy transfer between Cy3 and Cy5-labeled cholera toxin B subunit (CtxB), a pentavalent GM-1 probe. These experiments showed cholesterol-dependent clustering GM1/CtxB on the cell surface and a striking reduction in GM1 (-CtxB) from clathrin-coated pits (12). In contrast, when Ben performed FRET analysis of fluorescent citrin and CFP$\mathrm{GPI}$-anchored proteins, he obtained no evidence for a lipid-dependent clustering between the two GPI-anchored proteins. The discrepancy between the failure to detect lipid-mediated cohesion between GPI-anchored proteins in these experiments as well as earlier analyses and the clustering detected in Jitu Mayor's experiments may be reconciled by the higher sensitivity of anisotropy measurements in Mayor's homotypic FRET set-up.

Further evidence for the loose coupling between noncross-linked GPl-anchored proteins was presented by Gerhard Schuetz (Linz), who studied, using video microscopy, trajectories of single $\mathrm{GPl}$-anchored proteins in T lymphocytes.

Tracking single molecule behavior in cell membranes has led Akihiro Kusumi (Nagoya) to the description of compartmentalization of the plasma membrane into 30-200-nm compartments in which membrane proteins and lipids are trapped in time-scales between a millisecond to one hundred milliseconds (depending on the cell type and molecules). The majority of membrane molecules undergo 'hopping' from one compartment to the next. The compartment boundaries for membrane molecules are believed to consist of a proteinaceous 'picket fence' made of the membrane skeleton as well as the transmembrane proteins anchored to the membrane skeleton (13). Using video microscopy, Akihiro Kusumi followed diffusion rates of a GPI-anchored protein (CD59), a typical raft protein, and the non-raft lipid dioleoyl-PE. Both raft and non-raft markers undergo hop diffusion over membrane compartments at very similar hop rates (and therefore very similar macroscopic diffusion rates), showing that, in rest- ing steady-state, GPI-anchored proteins do not make prolonged associations with large and stable rafts (14). Aki Kusumi showed that the situation dramatically changes upon cross-linking or liganding of GPI-anchored proteins, e.g. using colloidal gold particles coated with multiple antibodies: this leads to the formation of large, stabilized rafts. These enlarged, stabilized rafts still undergo hop diffusion at a rate much slower than the GPI-anchored protein at steady-state. In addition, they exhibited transient immobilization which depends on cholesterol, actin, and tyrosine phosphorylation. During the short immobilization periods, phospholipase $\mathrm{C}$ is temporarily recruited, producing IP3, suggesting that these rafts function as the platform for transducing the CD59 signal to the intracellular calcium signal. This highlights the small, unstable nature of the rafts (plasma membrane phases) in the steady-state cell, and shows that clustering of raft markers generates domains which function as signaling centers.

The previous presentations addressed the organization of outer leaflet membrane lipids and GPI-anchored proteins. Rob Parton (Brisbane) presented clear indications of a domain structure in the inner lipid leaflet of the plasma membrane. These were obtained by analyzing the distribution of inner leaflet plasma markers using immuno-electron microscopy of isolated plasma membrane sheets. These studies utilized markers inserted via the H-ras (palmitoylation, farnesylation) or K-ras derived (polybasic stretch farnesylation) membrane anchor. Statistical analysis showed a partitioning of the two respective membrane markers into distinct domains of the plasma membrane (15). Moreover, Rob Parton showed coupling of the $\mathrm{H}$ ras-anchored marker with clusters of GPI-anchored proteins in the outer plasma membrane leaflet generated by antibody cross-linking.

Inner-outer leaflet coupling was also presented by Antonella Viola (Padova), who used cyan fluorescent protein with a myristoyl palmitoyl membrane anchor as an inner leaflet raft marker to monitor the distribution of inner leaflet rafts in resting $\mathrm{T}$ lymphocytes. This marker colocalized with clustered GPI-anchored proteins in a cholesterol-dependent fashion, providing further support for the concept of bilayer coupling. Interestingly, colocalization of myr palm CFP with domains formed by cross-linking of TCR complex appears independent of cholesterol.

Sandor Damjanovich (Debrecen) has established the analysis of membrane protein clusters using labeling with fluorescent antibodies and measurement of energy transfer between the probes. In his presentation he showed clustering of the TCR and potassium channel Kv1.3, using confocal microscopy, electron microscopy, fluorescence and energy transfer analysis (16). He discussed the small size of raft domains, the transient interactions of raft domains with lipid-shelled proteins and/or shallow differences between phases in the outer leaflet of the plasma membrane which make the detection of raft membrane domains in the plasma 
membrane's outer leaflet so difficult. Defined membrane domains were detected with inner leaflet anchored membrane markers. Clearly, it requires protein-mediated cross-linking of raft components to generate platforms for membrane-associated functions.

\section{Proteins Organizing Membrane Domains}

The analyses of raft markers in the plasma membrane showed that rafts are too small and raft lipid-mediated forces are not strong and specific enough to organize functional protein assemblies. In order to generate platforms for biological processes, additional protein scaffolds to stabilize interactions have to enter into play.

Prototypic functional raft membrane domains are the caveolae, which are organized by oligomers of the caveolin proteins. Caveolae are flask-shaped invaginations in the plasma membrane, and function as platforms for signaling events and endocytosis of specific molecules (17). Debbie Brown (Stony Brook) presented reconstitution of recombinant caveolin-1 into liposomes and analyzed its partitioning into detergent-resistant membranes. She showed that changes in caveolin 1's scaffolding domain, hydrophobic domain, and C-terminal domain all reduced DRM association, but that palmitoylation was not required for DRM association. Upon overexpression in cells, caveolin variants, which are deficient in DRM association, preferentially accumulated in the Golgi region, while DRM-associated variants were targeted to the plasma membrane, suggesting a role for raft association in transport through the Golgi apparatus.

The palmitoylated protein flotillin is another DRMassociated molecule with a possible role in organizing membrane domains. Flotillin has previously been implicated in insulin receptor signaling, where it was proposed to form a complex with CAP and Cbl. Ritva Tikkanen (Frankfurt) showed that flotillin/reggie-1 overexpression in fibroblasts induces actin filopodia. Possibly it plays a role in actin organization.

Jean Gruenberg presented the role of annexin II in defining membrane domains in the endocytic membrane. Annexin II belongs to a family of lipid binding proteins and is associated with early endosomal membranes in a cholesterol-dependent fashion. Interestingly, in fibroblasts of Niemann-Pick C (NPC) patients, annexin II localizes at late endosome. This coincides with the accumulation of cholesterol in late endosomes of NPC cells. Moreover, he presented data showing that RNAi-mediated block of annexin II expression inhibited budding of multivesicular transport vesicles from early endosomes. These data suggested that annexin $\|$ is responsible for defining cholesterol-rich membrane domains on early endosomes that segregate from multivesicular portions destined to become multivesicular carrier vesicles for transport to the late endosome (18).
The Golgi-associated protein GREG may be an exciting new organizer of membrane domains in the Golgi apparatus. Bernd Helms (Utrecht) showed that GREG interestingly combines two features: a coiled coil region and a GPI anchor. He found that the protein is an important regulator of Golgi structure. Hence, one may speculate that, via the coiled regions, the protein may have a propensity to oligomerize defining membrane domains in the Golgi membranes.

Klaus Fiedler (Basel) presented a proteomic analysis of immunoisolated caveolae from endothelial cells, suggesting a link to the actin cytoskeleton. He showed that a significant amount of transendothelial transport of albumin occurs via paracellular leakage.

\section{Rafts in Signaling: Making Use of the Elastic System}

Signal transduction via cell surface receptors is believed to utilize lateral compartmentalization into dynamic membrane domains in order to trigger receptor signaling cascades, organize receptor complexes, and to serve as platforms for the assembly of multimolecular signal transduction machineries and the integration of costimulatory and accessory signals.

The involvement of membrane domains in signaling via immunoreceptors (IRs) has been implicated in two distinct phases (Figure 2): first upon ligand binding, the acquisition and/or coalescence of ordered membrane phases in the receptor membrane environment has been suggested to allow efficient interaction (and activation) of raft associated src related kinases. This first phase may be a mainly biophysical lipid-mediated process. The second phase encompasses the signaling dependent assembly of a multimolecular protein signaling machinery, leading to stabilization of receptors in detergent-resistant membranes, a phenomenon mainly driven by protein-protein interactions $(19,20)$.

These two phases were addressed by Susan Pierce (Rockville), studying B lymphocyte activation by triggering BCR (B-cell antigen receptor). She presented important evidence for the acquisition of an ordered raft-like membrane environment in the first stage of BCR signaling using the ordered membrane staining DilC16 fluorescent lipid dye for energy transfer with BCR-associated fluorescence probe. Susan also presented data showing that a costimulatory signal via complement receptor CD21/CD19 complexes prolonged the lifetime of tyrosine phosphorylations in DRMs, probably extending the time of active signaling. Lastly, she described the presence of a palmitoylated tetraspanin molecule CD81 in the BCR-CD19/ CD21 complexes, which may further augment BCR CD19/CD21 costimulation. This suggests that raft association of BCR is composed of contributions of a first (signaling 


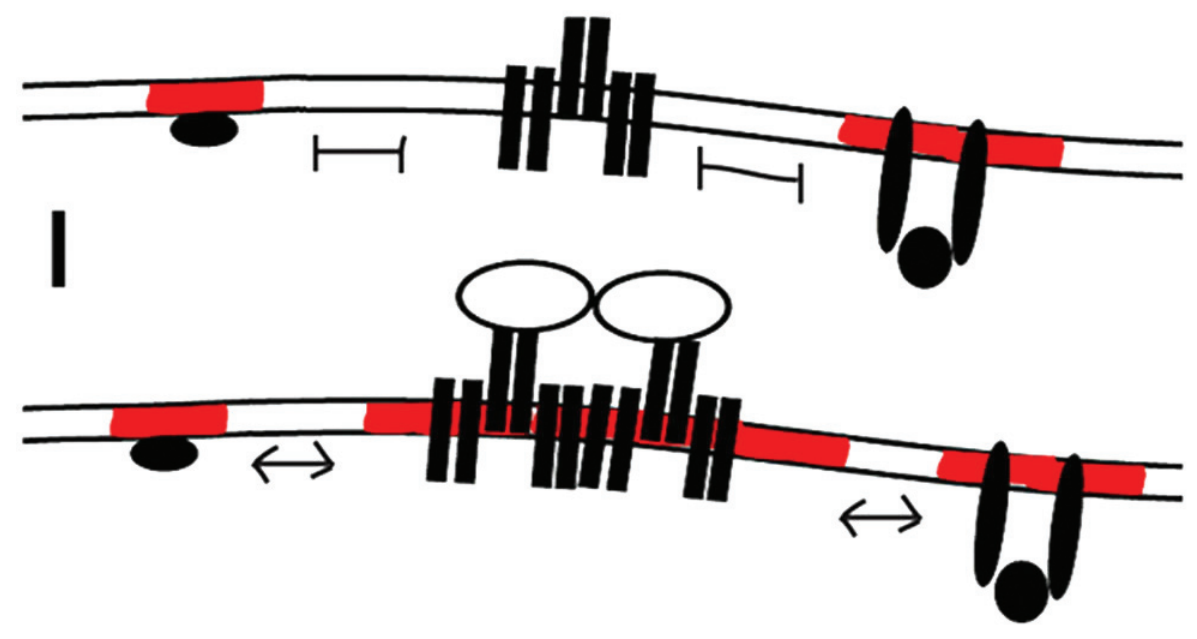

Figure 2: Model of two phases of raft membrane domains involvement in immune receptor (IR) signaling. (I) Induced phase miscibility: Engagement of IR may trigger transition of its membrane environment to form an ordered membrane-phase miscible with membrane environment of DRM-associated myristoylated and palmitoylated Src-kinases. Facilitated access of raft-associated Src-kinases to engaged IR leads to increased phosphorylation. An increase in the size of the raft domains around the IR may also increase the efficiency. (II) Formation of multimolecular signaling assemblies by protein/ protein interactions such as $\mathrm{SH} 2 /$ $\mathrm{SH} 3$-mediated interactions. These complexes are stably anchored in detergent-resistant membranes. Adapted with permission from (38).

independent phase) acquisition of an ordered membrane environment followed by the second (signaling dependent) formation of multiprotein signaling machinery which stabilizes DRM association.

Barbara Baird (Ithaca) studied how engagement of $\lg E$ receptors couples to activation of Lyn src related kinase and activates signaling in mast cells. She suggested an alternative flavor for phase 1 of IR signaling, namely that clustering of IgE receptors (FceRI) by antigen causes coalescence of FceRl with active Lyn in lipid rafts. This raft environment promotes Lyn activity by protecting Lyn activation site Tyr 397 from dephosphorylation. In $\mathrm{CHO}$ cells, expressed Lyn is highly active in a constitutive fashion, resulting in a high level of basal phosphorylation of FceRl. Co-expression of the transmembrane tyrosine phosphatase PTPalpha (21) reconstitutes antigen-dependent phosphorylation of FceRI. A raft-associating variant of PTPalpha suppresses Lyn activity but fails to reconstitute antigen-dependent signaling. Also, Petr Draber (Prague) studied membrane domains in mast cells. He reported that when Lyn colocalized with aggregated FceRI, there was no colocalization of Lyn with aggregated GPI-anchored protein Thy-1. Cholesterol depletion induced tyrosine phosphorylation of several substrates and markedly enhanced secretory responses elicited by cross-linking of both FceRI and Thy-1. Moreover, he presented data indicating that preincubation of cells with GM1 and other gangliosides did not inhibit tyrosine phosphorylation of FceRI and LAT adaptor, but inhibited tyrosine phosphorylation of PLC $\gamma$, and all subsequent events in IgE receptor signaling. Thy-1-mediated secretory response in ganglioside-pretreated cells was not affected.

Hai-Tao He (Marseille) introduced detergent Brij 98 resistant membranes from mouse $T$ cells. This detergent leaves raft markers insoluble even at $37^{\circ} \mathrm{C}$, while solubilizing nonraft markers. He showed that these DRMs, prepared from mouse T cells, have the typical signature of TX100 DRMs concentrating GPI-anchored proteins and sphingolipids. Interestingly, these DRMs also contained TCR and active src-related kinases and raft-associated adaptors (22).

Using a detergent-free method to immunoisolate TCR (Tcell antigen receptor) signaling plasma membrane domains, Thomas Harder (Oxford) studied the multimolecular signaling machinery that forms following activation of the TCR. He showed that the formation of these dynamic signaling domains is driven by the formation of multiprotein complexes which form by cooperative assembly of multiple $\mathrm{SH} 2 \mathrm{SH} 3$ adaptors and phospholipase $\mathrm{C}$ at the site of TCR triggering (23).

Antonella Viola (Padova) showed that a CFP-inner leaflet raft marker accumulated in the contact zone of resting human $\mathrm{T}$ cells to antigen-presenting cells. This was only 
observed upon coengagement of the TCR together with CD28 costimulatory receptor. She suggested that only CD28 costimulation involved rearrangement of raft membranes, while TCR signaling alone is not associated with raft rearrangement. Indeed, she showed that in her resting T-cell system tyrosine phosphorylation elicited by TCR engagement alone was insensitive to cholesterol extraction. This may also relate to her observation that colocalization of the TCR-CD3 complex with myr/palm-CFP was insensitive to cholesterol depletion, suggesting that ordered membrane domains in the vicinity of antibody cross-linked TCR do not require cholesterol.

EGF receptor and PDGF receptor tyrosine kinases are likewise organized in specific lateral assemblies. Paul van

Bergen en Henegouwen (Utrecht) characterized the lipid requirements for the formation of the high-affinity dimerized EGF receptor. By in vitro reconstitution and by FLIM energy transfer measurements, he showed that the dimeric form strongly depended on the presence of cholesterol in the membrane. This showed an important example of interplay between lipid- and protein-mediated interactions to form functional receptor complexes. Janos Szollosi (Debrecen) presented oligomers formed by EGFR family members ErbB2 and ErbB3 using energy transfer measurements and scanning near-field optical microscopy. He showed two levels of lateral organization: first ErbB2 and 3 dimerization and, second, larger clusters with several hundred receptors which colocalized with CTxB-stained GM1 (24).

The small GTPase Rac is an important regulator of actin rearrangement following integrin signaling. Miguel del Pozo (La Jolla) presented targeting of GTP Rac into raft membrane domains. In order to study the relationship to rafts, he stimulated nonadherent cells with GM1 binding beads. Activated Rac concentrated at the contact regions of these beads. He showed that Integrins regulated lipid raft assembly in the plasma membrane via an effect on the protein caveolin-1. These observations provide a way to understand mechanisms by which signaling centers generated by cross-linked raft domains couple to the actin cytoskeleton.

Bice Chini (Milano) presented an interesting indication of raft domains regulating the coupling of seven-span receptors to heterotrimeric G-proteins. She studied the effects of targeting the seven-span Oxytocin receptor (OTR) into DRM domains by fusing it to caveolin 2. The OTR couples to $\mathrm{G} \alpha \mathrm{q} / 11$ and mediates an inhibitory effect on proliferation, which is sensitive to pertussis toxin treatment. Interestingly, the cav2 chimeric receptor induced a proliferation in response to oxytocin treatment. The proliferative response transduced by OTR-cav2 was not sensitive to pertussis toxin, suggesting that distinct effects of oxytocin stimulation via WT and OTR-cav2 were caused by receptor coupling to different heterotrimeric $\mathrm{G}$ proteins (25).

Agnese Mariotti (Lausanne) studied the correlation between the progression in malignancy of melanoma cells and the partitioning of integrins and $\mathrm{N}$-cadherin into DRMs. Malignant radial growth phase (RGP) melanoma cells and more aggressive vertical growth phase (VGP) melanoma cells exhibited differential partitioning of integrins and $\mathrm{N}$-cadherin into DRMs.

Richard Anderson (Dallas) showed that signaling cascades via PDGF receptors lead to MAPK activation in a caveolar membrane fraction. He reported that cholesterol depletion causes activation of Erk-1 and 2 and presented an explanation for this, at first sight, paradoxical observation: his group succeeded in purifying a large cytoplasmic Erk phosphatase complex containing PP2A and HePTP. The integrity of this complex depended on the presence of cholesterol, providing an explanation of why cholesterol depletion induced the activation of MAP kinases (26).

\section{Rafts in Pathology}

Internalization, intracellular trafficking, and processing of pathogens have been tightly linked to raft domains. Rafts are thought to help binding of multimeric toxins, to serve as concentration devices to promote oligomerization, and may function as platforms for pathogen internalization and budding.

In her EMBO Young Investigator Lecture, Gisou van der Goot (Geneva) described how different bacterial toxins have evolved to attack cells by binding raft components. Anthrax toxin contains three subunits: the protective antigen (PA), the metalloprotease lethal factor (LF) and the edema factor (EF). LF, which cleaves MAPKinases, and EF, an adenylate cyclase, have to enter the cell's cytoplasm in order to exert their toxic function. This is initiated by an internalization step that is mediated by PA. PA binds to the anthrax toxin receptor (ATR) and is cleaved by furin, leading to the formation of a PA heptamer. This forces heptamerization of ATR, which triggers its DRM targeting and endocytosis. The DRM translocation upon clustering may resemble phase 1 of IR triggering (Figure 1). The internalization of the ATR occurs via clathrin-coated structures independently of caveolae, by a dynamin/eps 15 dependent process (27).

Ludger Johannes (Institut Curie, Paris) analyzed internalization of Shiga toxin via its receptor glycosphingolipid Gb3/ $\mathrm{CD} 77$, and followed the steps of its retrograde transport via the early endosomes and the Golgi into the ER. Transport from the early endosome to the TGN occurs via DRMs, probably involving VAMP-3 and -4 SNARE microdomains (28). Ludger moreover focused on clathrin-coated-vesiclemediated membrane sorting in Shiga toxin transport. He presented the down-modulation of clathrin expression by siRNA and the analysis of clathrin distribution on wholemount specimens. Together with data obtained by a permeabilized cell assay for Shiga toxin transport to the TGN, a connection is drawn between clathrin function at several levels in the cell and retrograde transport. 
Albert Taraboulos (Jerusalem) presented the involvement of raft membrane domains in the formation of prions, the causative agents of transmissible spongiform encephalitis. Both isoforms of the prion protein, $\operatorname{PrP}^{\mathrm{C}}$ and $\mathrm{PrP}^{\mathrm{Sc}}$, have a GPI anchor, and thus both are in rafts. It is believed that the pathogenic $\mathrm{PrP}^{\mathrm{Sc}}$ protein forces the conversion of the nonpathogenic $\operatorname{PrP}^{C}$ into the toxic $\beta$-sheet conformation of $\operatorname{PrP}^{\mathrm{Sc}}$. $\mathrm{PrP}^{\mathrm{C}}$ can be patched by cross-linking. Interestingly, in the ScNa neuroblastoma cell system, the conversion of $\operatorname{PrP}^{\mathrm{C}}$ to $\mathrm{PrP}^{\mathrm{Sc}}$ was sensitive to cholesterol depletion but was stimulated by sphingolipid down-modulation by sphingomyelinase or fumonisin treatments. PrPC DRMs and $\operatorname{PrP}^{\mathrm{Sc}}$ DRMs floated to different densities in a gradient centrifugation; hence, Albert suggested that these proteins associate primarily with different types of raft membrane domains. Albert presented the attractive idea that the conversion is facilitated by approximation of the prion protein isoforms in membrane microdomains.

Cells internalize SV40 virus via caveolae. In his talk, Ari Helenius (Zürich) suggested that after binding of the virus to the cell surface the virus is trapped in caveolae, leading to a cascade of signaling events. This triggers dynamin-, actinand cholesterol-dependent virus internalization. The internalized virus moves via the caveosome compartment to the ER. The sorting mechanisms are under investigation. Interestingly, SV40 causes recruitment of GFP Arf1 to the caveosome, possibly indicating a COP1 involvement in the retrograde transport. Moreover, overexpression of a constitutively active variant of rab5 trapped the virus in the early endosome. Ari showed that SV40 is also internalized in cells from caveolin-1 knock-out mice. Here, the internalization may follow a different molecular mechanism, as it was insensitive to actin disruption and dynamin dominant negative mutants. It appears that virus internalization involved a caveosome-like compartment in the cav-1 knock-out cells.

\section{Lipid Transport and Cholesterol}

Sterols, such as cholesterol in animal cells and ergosterol in yeast, are essential elements in membrane organization.

Teymuras Kurzchalia (Dresden) presented Caenorhabditis elegans as an amazing sterol auxotroph organism which appears to get along with very low amounts of sterol lipids. Observing a phenotype caused by starving $C$. elegans of cholesterol required some effort to remove residual sterols out of the propagation system. Only after one generation was the ergosterol-starving phenotype, growth arrest, observed - caused by its inability to shed its cuticle at the L2 stage of larval development. This is most likely due to an endocrine problem caused by the lack of a steroid hormone for which the search is ongoing. But how do the worms survive without sphingolipid cholesterol raft domains? Possibly they compensate the defect by branched side chains on their sphingolipids allowing the necessary fluidity of their membranes.
Elina Ikonen (Helsinki) studied efflux of cholesterol biosynthetic precursors using serum and methyl $\beta$-cyclodextrin as acceptors. She found that lathosterol and particularly zymosterol precursors were more efficient in the efflux from cells than cholesterol. Moreover, the newly synthesized precursor lathosterol was more rapidly incorporated into DRMs (29). She presented the lipids of virus particles from fibroblasts of patients with a Niemann-Pick $\mathrm{C}$ (NPC) cholesterol storage disease. In Influenza and Vesicular Stomatitis Virus virions propagated in NPC fibroblasts, cholesterol and also polyunsaturated fatty acids were enriched, possibly representing a compensatory mechanism to ensure proper membrane fluidity (30).

\section{Membrane Sorting and Polarity}

One of the earliest sprouts of the raft hypothesis was the concept of glycolipid assemblies forming the platform for apical transport and polarization of epithelial cells.

Again, yeast genetics provides extremely powerful tools to dissect principles of raft lipid organization, its effect on membrane sorting/transport, and finally cell polarity. Roger Schneiter (Fribourg) went into the oily part of the question by studying the genetic interaction of a mutant deficient in the last elongation step of the fatty acid of ceramide generating a C26 chain. Interestingly, this mutant was lethal in conjunction with a deficiency in adding a specific methyl group to ergosterol, the yeast sterol lipid analogous to the cholesterol in mammalian cells. This provides important evidence for the highly specific interactions of sterols and sphingolipids. Moreover, this double mutant exhibited a specific defect in DRM association of the plasma membrane $\mathrm{H}+$ ATPase, leading to its missorting and degradation in the vacuole (31).

Work of Howard Riezman's (Geneva) group has shown that the GPI-anchored protein, Gas1p, segregates into distinct carriers upon exit from the endoplasmic reticulum (ER), and that this mechanism depends upon previously described tethering factors and SNAREs. He showed that stable association of Gas1p with ER membranes requires ceramide synthesis (32).

Mating signals generate the polarized 'shmoo' morphology in the Saccharomyces cerevisiae yeast cell. Kai Simons (Dresden) showed that GPI-anchored proteins concentrate in the polar surface projection of the shmoos and that mutants of raft lipid sphingolipid and ergosterol synthesis are defective in shmoo formation and mating (33). Clearly, yeast genetics will continue to help unravel the complex interplay between raft lipid- and protein-mediated organization of membrane sorting and cell polarity. Following this line, Kai Simons discussed the role of glycosylations in yeast in biosynthetic membrane transport and polarity, testing the hypothesis that glycosylated proteins interact with 
lectins to organize membrane subdomains as platforms for sorting.

The picture becomes more complicated in animal cells. Carlos Martinez-A (Madrid) put forward a concept of selectively polarized $U$ and $L$ rafts in the migrating $T$ cell: $T$ lymphocytes migrate in response to chemotactic stimuli and exhibit an intriguing segregation of raft markers into the leading edge ( $L$ rafts concentrating chemokine receptors, GM-3) and the uropod (U rafts concentrating ICAM-1 and $(\mathrm{GM}-1$ ) at the opposite poles of the cells (34). He provided evidence for an enhanced formation of $D 3,4,5$ phosphorylated $\mathrm{PI}$ in the leading edge and suggested that this gradient drives a polarization process via myosin/actin driven process.

Chiara Zurzolo (Naples) provided evidence for a clustering dependent sorting of $\mathrm{GPI}$-anchored proteins in polarized epithelial Fisher Rat Thyroid (FRT) cells. In FRT cells, different GPI-anchored proteins are differentially sorted, showing that the GPI anchor is not a sufficient apical targeting signal. GPI-anchored placental alkaline phosphatase (PLAP) is targeted to the apical membrane in FRT cells. Upon biosynthetic pulse, PLAP becomes TX-100 insoluble and forms high molecular weight complexes (HMWC), in contrast to basolaterally sorted GPI-anchored proteins that are not able to form HMWC, although TX-100 insoluble.

Richard Pagano (Rochester) summarized the evidence for the caveolar endocytosis of fluorescent glycosphingolipid (GSL) analogs, including BODIPY-Lactosylceramide (LacCer), in multiple cell types. Data were also presented showing that treatment of cells with exogenous GSLs (natural or synthetic) can selectively stimulate internalization through caveolae. Deepak Sharma, from Pagano's group, presented a Poster using the monomer-excimer properties of BODIPY-LacCer to study the formation of GSL microdomains in early endosomes of living cells (35).

George Banting (Bristol) described the protein BST-2/ $\mathrm{HM} 1.24$, and demonstrated that this protein contains two modes of membrane anchorage: a transmembrane region and a GPI anchor (36). This protein cycles between the cell surface and intracellular location (probably the TGN) is delivered to the apical membrane of hepatocytes and is apparently internalized via both clathrin-dependent and clathrin-independent pathways.

In her presentation, Angela Wandinger-Ness (Albuquerque) discussed the role of rab7 in the regulation of late endosomal membrane microdomains that may be important for invagination of multivesicular late endosomes. Internalized EGFR and MHC class II molecules in $\mathrm{MHC}$ class II presenting cells localize to these internal structures. hVps34 is a phosphatidylinositol (PI) 3 kinase which catalyses formation of PI 3 phosphate, a lipid species which recruits proteins carrying FYVE domains to early endosomal membranes. On early endosomes, hVps34 is in a complex with Rab5 and p150. It is known that antibodies to hVps34 inhibit the internalization of EGFR into multivesicular endosomes (37). Angela addressed the role rab7 in targeting and function of vps34 on late endosomes, providing evidence for a complex between rab7, hVps34 and p150. Overexpression of nucleotide free rab7 inhibited hVPS34 kinase activity on late endosomes and reduced the level of internal membranes in rab7-positive endosomes.

Koret Hirschberg (Tel Aviv) utilized photoactivated GFP to follow movement and segregation of GFP-tagged cargo molecules through the Golgi apparatus. He compared the biosynthetic transport route of VSV-G, collagen GFP and signal sequence-GFP. Measurement of loss of fluorescence from the unbleached Golgi apparatus, or, alternatively, from photoactivated-GFP tagged cargo in the Golgi, show that the markers rapidly equilibrate within the Golgi. This equilibration was observed also upon depletion of ATP. His data suggested that the Golgi is one compartment. However, ATP depletion abolished the time-dependent segregation of the VSV-G cargo from Golgi-resident marker GalT.

\section{Conclusions}

The physical term of membrane phases can clearly be applied to biological membranes; however, this meeting has clearly shown that the liquid ordered/liquid disordered biphasic systems in artificial membranes are not sufficient to describe lateral compartmentalization of cell membranes. A large number of proteins were presented which will scaffold protein assemblies and organize functional membrane domains, and the next phase most likely will require understanding of the organizing principles for each biological activity associated to a membrane domain.

\section{Acknowledgments}

We are grateful for the support provided to the meetings by the European Commission, Research DG, Human Potential Programme, High-Level Scientific Conferences, Contract HPCF-CT-2000-00180, by EMBO and by the Klaus Tschira Foundation.

This paper is based on presentations at the Euresco Conference/EMBO Workshop on Microdomains, Lipid Rafts and Caveolae and a subsequent conference in honor of Kai Simons in Tomar, Portugal, May 17-25, 2003. The Euroconference was organized by the European Science Foundation. This information is the sole responsibility of the authors.

\section{References}

1. Simons K, Ikonen E. Functional rafts in cell membranes. Nature 1997;387:569-572 


\section{Harder et al.}

2. Brown DA, London E. Structure of detergent-resistant membrane domains: does phase separation occur in biological membranes? Biochem Biophys Res Commun 1997;240:1-7.

3. McConnell HM, Vrljic M. Liquid-liquid immiscibility in membranes. Annu Rev Biophys Biomol Struct 2003;32:469-492.

4. Edidin M. The state of lipid rafts: from model membranes to cells. Annu Rev Biophys Biomol Struct 2003;32:257-283.

5. Huang J, Feigenson GW. A microscopic interaction model of maximum solubility of cholesterol in lipid bilayers. Biophys J 1999;76: 2142-2157.

6. Sarasij RC, Rao M. Tilt texture domains on a membrane and chirality induced budding. Phys Rev Lett 2002;88:88-101.

7. Lipowsky R. Domains and rafts in membranes - hidden dimensions of self organization. J Biol Phys 2002;28:195-210.

8. Pomorski T, Lombardi R, Riezman H, Devaux PF, Van Meer G, Holthuis JC. Drs2p-related P-type ATPases Dnf1p and Dnf2p are required for phospholipid translocation across the yeast plasma membrane and serve a role in endocytosis. Mol Biol Cell 2003;14:1240-1254.

9. Khan TK, Yang B, Thompson NL, Maekawa S, Epand RM, Jacobson K. Binding of NAP-22, a calmodulin-binding neuronal protein, to raft-like domains in model membranes. Biochemistry 2003;42:4780-4786.

10. Anderson RG, Jacobson K. A role for lipid shells in targeting proteins to caveolae, rafts, and other lipid domains. Science, 2002;296: 1821-1825

11. Varma R, Mayor S. GPI-anchored proteins are organized in submicron domains at the cell surface. Nature 1998;394:798-801.

12. Nichols BJ. GM1-containing lipid rafts are depleted within clathrincoated pits. Curr Biol 2003;13:686-690.

13. Nakada C, Ritchie K, Oba Y, Nakamura M, Hotta $Y$, lino R, Kasai RS, Yamaguchi K, Fujiwara T, Kusumi A. Accumulation of anchored proteins forms membrane diffusion barriers during neuronal polarization. Nat Cell Biol 2003;5:626-632.

14. Fujiwara T, Ritchie K, Murakoshi H, Jacobson K, Kusumi A. Phospholipids undergo hop diffusion in compartmentalized cell membrane. J Cell Biol 2002;157:1071-1081.

15. Prior IA, Muncke C, Parton RG, Hancock JF. Direct visualization of Ras proteins in spatially distinct cell surface microdomains. J Cell Biol, 2003;160:165-170.

16. Panyi G, Bagdany M, Bodnar A, Vamosi G, Szentesi G, Jenei A, Matyus L, Varga S, Waldmann TA, Gaspar R, Damjanovich S. Colocalization and nonrandom distribution of Kv1.3 potassium channels and CD3 molecules in the plasma membrane of human T lymphocytes. Proc Natl Acad Sci USA 2003;100:2592-2597.

17. Liu P, Rudick M, Anderson RG. Multiple functions of caveolin-1. J Biol Chem 2002;277:41295-41298.

18. Mayran N, Parton RG, Gruenberg J. Annexin II regulates multivesicular endosome biogenesis in the degradation pathway of animal cells. EMBO J 2003;22:3242-3253.

19. Dykstra M, Cherukuri A, Sohn HW, Tzeng SJ, Pierce SK. Location is everything: lipid rafts and immune cell signaling. Annu Rev Immunol 2003;21:457-481.

20. Harder T. Raft membrane domains and immunoreceptor functions. Adv Immunol 2001;77:45-92.

21. Young RM, Holowka D, Baird B. A lipid raft environment enhances lyn kinase activity by protecting the active site tyrosine from dephosphorylation. J Biol Chem 2003;278:20746-20752.

22. Drevot $P$, Langlet $C$, Guo XJ, Bernard AM, Colard O, Chauvin JP, Lasserre R, He HT. TCR signal initiation machinery is pre-assembled and activated in a subset of membrane rafts. EMBO J 2002;21: 1899-1908.

23. Hartgroves LC, Lin J, Langen H, Zech T, Weiss A, Harder T. Synergistic assembly of linker for activation of $T$ cells signaling protein complexes in T cell plasma membrane domains. J Biol Chem 2003;278:20389-20394.

24. Nagy P, Vereb G, Sebestyen Z, Horvath G, Lockett SJ, Damjanovich S, Park JW, Jovin TM, Szollosi J. Lipid rafts and the local density of ErbB proteins influence the biological role of homo- and heteroassociations of ErbB2. J Cell Sci 2002;115:4251-4262.

25. Rimoldi V, Reversi A, Taverna E, Rosa P, Francolini M, Cassoni P, Parenti M, Chini B. Oxytocin receptor elicits different EGFR/MAPK activation patterns depending on its localisation in caveolin-1 enriched domains. Oncogene 2003; in press.

26. Wang PY, Liu P, Weng J, Sontag E, Anderson RG. A cholesterolregulated PP2A/HePTP complex with dual specificity ERK1/2 phosphatase activity. EMBO J 2003;22:2658-2667.

27. Abrami L, Liu S, Cosson P, Leppla SH, van der Goot FG. Anthrax toxin triggers endocytosis of its receptor via a lipid raft-mediated clathrindependent process. J Cell Biol 2003;160:321-328.

28. Falguieres T, Mallard F, Baron C, Hanau D, Lingwood C, Goud B, Salamero J, Johannes L. Targeting of Shiga toxin B-subunit to retrograde transport route in association with detergent-resistant membranes. Mol Biol Cell 2001;12:2453-2468.

29. Lusa S, Heino S, Ikonen E. Differential mobilization of newly synthesized cholesterol and biosynthetic sterol precursors from cells. J Biol Chem 2003;278:19844-19851.

30. Blom TS, Koivusalo M, Kuismanen E, Kostiainen R, Somerharju P, Ikonen E. Mass spectrometric analysis reveals an increase in plasma membrane polyunsaturated phospholipid species upon cellular cholesterol loading. Biochemistry 2001;40:14635-14644.

31. Eisenkolb M, Zenzmaier C, Leitner E, Schneiter RA Specific structural requirement for ergosterol in long-chain fatty acid synthesis mutants important for maintaining raft domains in yeast. Mol Biol Cell 2002;13:4414-4428.

32. Watanabe $R$, Funato $K$, Venkataraman $K$, Futerman $A H$, Riezman $H$ Sphingolipids are required for the stable membrane association of glycosylphosphatidylinositol-anchored proteins in yeast. J Biol Chem 2002;277:49538-49544.

33. Bagnat $M$, Simons $K$. Cell surface polarization during yeast mating. Proc Natl Acad Sci USA 2002;99:14183-14188.

34. Gomez-Mouton C, Abad JL, Mira E, Lacalle RA, Gallardo E, JimenezBaranda S, Illa I, Bernad A, Manes S, Martinez-A C. Segregation of leading-edge and uropod components into specific lipid rafts during T cell polarization. Proc Natl Acad Sci USA 2001;98:9642-9647.

35. Sharma DK, Choudhury A, Singh RD, Wheatley CL, Marks DL, Pagano RE. Glycosphingolipids internalized via caveolar-related endocytosis rapidly merge with the clathrin pathway in early endosomes and form microdomains for recycling. J Biol Chem 2003;278:7564-7572.

36. Kupzig S, Korolchuk V, Rollason R, Sugden A, Wilde A, Banting G. BST$2 / \mathrm{HM} 1.24$ is a raft-associated apical membrane protein with an unusual topoloy. Traffic 2003;4:694-704.

37. Futter CE, Collinson LM, Backer JM, Hopkins CR. Human VPS34 is required for internal vesicle formation within multivesicular endosomes. J Cell Biol 2001;155:1251-1264.

38. Harder T. Formation of functional cell membrane domains: the interplay of lipid- and protein-mediated interactions. Philos Trans R Soc Lond B Biol Sci 2003;358:863-868. 